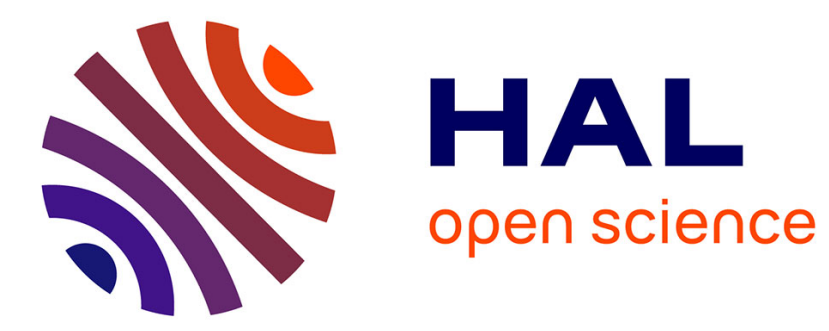

\title{
A macroscopic model for platooning in highway traffic
}

Giulia Piacentini, Paola Goatin, Antonella Ferrara

\section{To cite this version:}

Giulia Piacentini, Paola Goatin, Antonella Ferrara. A macroscopic model for platooning in highway traffic. SIAM Journal on Applied Mathematics, 2020, 80 (1), pp.639-656. hal-02309950

\section{HAL Id: hal-02309950 \\ https://hal.science/hal-02309950}

Submitted on 9 Oct 2019

HAL is a multi-disciplinary open access archive for the deposit and dissemination of scientific research documents, whether they are published or not. The documents may come from teaching and research institutions in France or abroad, or from public or private research centers.
L'archive ouverte pluridisciplinaire HAL, est destinée au dépôt et à la diffusion de documents scientifiques de niveau recherche, publiés ou non, émanant des établissements d'enseignement et de recherche français ou étrangers, des laboratoires publics ou privés. 


\title{
A macroscopic model for platooning in highway traffic
}

\author{
Giulia Piacentini $^{1} \quad$ Paola Goatin $^{2} \quad$ Antonella Ferrara $^{1}$
}

October 9, 2019

\begin{abstract}
We consider a model describing the presence of a platoon of vehicles moving in the traffic flow. The model consists of a coupled PDE-ODE system describing the interaction between the platoon and the surrounding traffic flow. The scalar conservation law takes into account the main traffic evolution, while the ODEs describe the trajectories of the initial and final points of the platoon, whose length can vary in time. The presence of the platoon acts as a road capacity reduction, resulting in a space-time discontinuous flux function. We describe the solutions of Riemann problems and design a finite volume numerical scheme sharply capturing non-classical discontinuities. Some numerical tests are presented to show the effectiveness of the method.
\end{abstract}

2010 Mathematics Subject Classification: 35L65, 90B20, 82B21.

Keywords: Scalar conservation laws with space-time discontinuous flux; Traffic flow modeling; PDE-ODE systems; conservative finite volume schemes.

\section{Introduction}

Macroscopic traffic flow models have known an increasing popularity in the last decades in the engineering and the applied mathematics literature, due to their capability of capturing traffic characteristics, their low computational cost and their relevance for solving optimal control problems [23, 32]. Yet, new technical advances affecting transportation dynamics, like routing devices and autonomous vehicles, are changing the current traffic characteristics, requiring the design of new models [12, 28, 29]. In particular, vehicle automation paves the way to truck platooning, intended to optimize freight transportation and to reduce fuel consumption [2, 18, 24, 33. Energy saving and eco-driving considerations can also encourage car platooning [35. In this paper, we aim at studying the interaction of a platoon of vehicles with the surrounding traffic flow. The platoon acts as an obstacle on the traffic flow reducing the capacity of the road in the occupied road portion. Several models considering slow moving vehicles acting on the flow as point moving bottlenecks have already been introduced in the literature [10, 11, 19, 21, 34, usually modeled as a time dependent flux constraint. Here, we extend the approach to take into account a platoon of vehicles having physical length, which may vary depending on the number of vehicles composing it and their spacing. We consider a macroscopic model consisting in a hyperbolic Partial Differential Equation (PDE) - the

\footnotetext{
${ }^{1}$ Department of Electrical, Computer and Biomedical Engineering, University of Pavia, Pavia, Italy. E-mail: giulia.piacentini02@universitadipavia.it, antonella.ferrara@unipv.it

${ }^{2}$ Université Côte d'Azur, Inria, CNRS, LJAD, France. E-mail: paola.goatin@inria.fr
} 
classical Lighthill-Whitham-Richards (LWR) equation [22, 27] - coupled with two Ordinary Differential Equations (ODEs) describing the trajectories of the front and back endpoints of the platoon. We are thus led to study a scalar conservation law with discontinuous flux in space and time, i.e. at the platoon endpoints, whose downstream velocity adapts to the traffic conditions.

The literature about conservation laws with discontinuous flux functions is very rich: while most of the research considers only discontinuities at fixed points in space (see for example [1] and references therein), few works deal with also time dependent discontinuities: we refer to [8, 17, 30, 31 and the very recent [4, 15. Even if the scalar conservation law in our model can be adapted to fit the much more general framework of [4], guaranteeing the existence and uniqueness of the vanishing viscosity limit solutions of the conservation law with predefined discontinuities, the strong coupling with the ODE trajectories makes the problem more involved. Moreover, in view of traffic modeling applications (see, for instance, those related to traffic control [7, 25, 26]), we are interested in providing a sharp maximum principle on the solutions and an efficient numerical strategy, able to precisely capture the density discontinuities arising at the platoon endpoints. To this end, we apply a conservative reconstruction strategy inspired by [3, 6], which requires the full knowledge of Riemann problem solutions at flux discontinuity points.

The paper is organized as follows. In Section 2, we introduce the PDE-ODE coupled model under consideration. The solutions to the corresponding Riemann problems of interest are detailed in Section 3. In Section 4, we describe the finite volume scheme developed to compute approximate solutions, which is tested in Section 5, proving its effectiveness.

\section{Mathematical model}

We adopt the macroscopic first order LWR model to describe the dynamics of the overall traffic flow. We denote by $z_{d}=z_{d}(t)$ and $z_{u}=z_{u}(t)$ respectively the downstream and upstream endpoints of the platoon. At the platoon location, the road capacity is reduced proportionally to the number of lanes occupied by the platoon, and the platoon acts as a flux constraint on the interval $\left[z_{u}(t), z_{d}(t)\right]$.

The resulting coupled PDE-ODE model reads

$$
\begin{array}{lr}
\partial_{t} \rho+\partial_{x} F(t, x, \rho)=0 & (t, x) \in \mathbb{R}^{+} \times \mathbb{R} \\
\rho(0, x)=\rho_{0}(x) & x \in \mathbb{R} \\
\dot{z}_{u}(t)=v_{u}\left(t, \rho\left(t, z_{u}(t)+\right)\right) & t \in \mathbb{R}^{+} \\
z_{u}(0)=z_{u}^{0} & \\
\dot{z}_{d}(t)=v_{d}\left(t, \rho\left(t, z_{d}(t)+\right)\right) & t \in \mathbb{R}^{+} \\
z_{d}(0)=z_{d}^{0} &
\end{array}
$$

Above, the conserved quantity $\rho=\rho(x, t)$ is the traffic density and the space-time discontinuous flux function $F$ is defined as

$$
F(t, x, \rho):= \begin{cases}f(\rho) & \text { if } x \notin\left[z_{u}(t), z_{d}(t)\right] \\ f_{\alpha}(\rho):=\alpha f(\rho / \alpha) & \text { if } x \in\left[z_{u}(t), z_{d}(t)\right] .\end{cases}
$$


In (2.2) we assume $f(\rho)=\rho v(\rho)$, and we restrict the present study to the linear speed-density relationship

$$
v(\rho)=V\left(1-\frac{\rho}{R}\right),
$$

even if more general speed laws could be considered. Above, the constant $V$ denotes the maximal speed of vehicles and $R$ is the maximal density reachable on the road, corresponding to a bumper-to-bumper situation, which is reduced to $\alpha R$ at the platoon position. Indeed, we have

$$
\begin{gathered}
f(\rho)=V \rho\left(1-\frac{\rho}{R}\right), \\
f_{\alpha}(\rho)=V \rho\left(1-\frac{\rho}{\alpha R}\right) .
\end{gathered}
$$

To comply with the varying road capacity, we have to consider initial data $\rho_{0}$ such that

$$
\begin{array}{ll}
\rho_{0}(x) \in[0, \alpha R] & \text { if } x \in\left[z_{u}^{0}, z_{d}^{0}\right], \\
\rho_{0}(x) \in[0, R] & \text { otherwise. }
\end{array}
$$

We remark that the points of maximum of $f$ and $f_{\alpha}$ are $\rho_{c r}=R / 2$ and $\rho_{c r}^{\alpha}=\alpha R / 2$ respectively, and we denote $f^{\max }=f\left(\rho_{c r}\right)$ and $f_{\alpha}^{\max }=f_{\alpha}\left(\rho_{c r}^{\alpha}\right)$.

The dynamics of the platoon ending points is governed by (2.1c)-(2.1e), where

$$
\begin{aligned}
& v_{u}(t, \rho):=\max \left\{V_{u}(t),-f_{\alpha}(\rho) /(R-\rho)\right\}, \\
& v_{d}(t, \rho):=\min \left\{V_{d}(t), v(\rho)\right\},
\end{aligned}
$$

where $V_{u}(t) \in[-V, V]$ and $V_{d}(t) \in[0, V]$ are the (controllable) maximal speeds of the upstream and downstream endpoints respectively. Equation (2.7) accounts for the fact that the platoon cannot move quicker than the downstream traffic velocity.

The length of the platoon $L(t):=z_{d}(t)-z_{u}(t)$ can vary depending on the spacing and the number of vehicles composing it. For a fixed number of vehicles, we assume $L_{\min } \leq L(t) \leq$ $L_{\max }$. Under these constraints, the length of the platoon varies according to

$$
\dot{L}(t)=\dot{z}_{d}(t)-\dot{z}_{u}(t)=v_{d}\left(\rho\left(t, z_{d}(t)+\right)\right)-v_{u}\left(\rho\left(t, z_{u}(t)+\right)\right) .
$$

Moreover, the speed $V_{d}$ is constrained to be positive, since vehicles cannot move backwards. On the other hand, if vehicles are allowed to join (and leave) the platoon, $V_{u}$ may take negative values. In the case of negative speed, condition (2.6) ensures that the problem is well posed, see Section 3.2 .

Following [17, Definition 5.1], we aim at constructing weak entropy solutions of (2.1) in the following sense:

Definition 2.1. A triple $\left(\rho, z_{u}, z_{d}\right) \in \mathbf{C}^{\mathbf{0}}\left(\mathbb{R}^{+} ; \mathbf{L}^{\mathbf{1}} \cap \mathbf{B V}(\mathbb{R} ;[0, R])\right) \times\left(\mathbf{W}^{\mathbf{1}, \infty}\left(\mathbb{R}^{+} ; \mathbb{R}\right)\right)^{2}$ is a weak entropy solution to (2.1) $-(2.2)-(2.5)$ if

(i) $\rho(t, x) \in[0, \alpha R]$ for a.e. $x \in\left[z_{u}(t), z_{d}(t)\right]$;

(ii) for all $\kappa \in \mathbb{R}$ and all test functions $\phi \in \mathbf{C}_{\mathbf{c}}^{\mathbf{1}}\left(\mathbb{R}^{2} ; \mathbb{R}^{+}\right)$it holds

$$
\int_{\mathbb{R}^{+}} \int_{\mathbb{R}}\left(|\rho-\kappa| \partial_{t} \phi+\operatorname{sgn}(\rho-\kappa)(F(t, x, \rho)-F(t, x, \kappa)) \partial_{x} \phi\right) d x d t
$$




$$
\begin{aligned}
& +\int_{\mathbb{R}}\left|\rho_{0}-\kappa\right| \phi(0, x) d x \\
& +\int_{\mathbb{R}^{+}}\left|F\left(t, z_{u}(t)+, \kappa\right)-F\left(t, z_{u}(t)-, \kappa\right)\right| \phi\left(t, z_{u}(t)\right) d t \\
& +\int_{\mathbb{R}^{+}}\left|F\left(t, z_{d}(t)+, \kappa\right)-F\left(t, z_{d}(t)-, \kappa\right)\right| \phi\left(t, z_{d}(t)\right) d t \geq 0 ;
\end{aligned}
$$

(iii) $z_{u}$ and $z_{d}$ are Carathéodory solutions of $(2.1 \mathrm{c})-(2.1 \mathrm{~d})$, respectively $(2.1 \mathrm{~d})-(2.1 \mathrm{f})$, i.e. for a.e. $t \in \mathbb{R}^{+}$it holds

$$
\begin{aligned}
& z_{u}(t)=z_{u}^{0}+\int_{0}^{t} v_{u}\left(s, \rho\left(s, z_{u}(s)+\right)\right) d s \\
& z_{d}(t)=z_{d}^{0}+\int_{0}^{t} v_{d}\left(s, \rho\left(s, z_{d}(s)+\right)\right) d s .
\end{aligned}
$$

Remark 2.2. (Maximum principle) To ensure a uniform bound on the solutions, related works in the literature usually assume that there exists an interval $[a, b]$ such that $F(t, x, a)=$ $F(t, x, b)=0$ for all $x \in \mathbb{R}, t \in \mathbb{R}^{+}$, see e.g. [4, 17]. Even if we can easily cope with this assumption setting $f_{\alpha}(\rho)=0$ for $\rho>\alpha R$, this would give a rough bound $\rho(t, x) \in[0, R]$ for the solutions of (2.1) $-(2.2)-(2.5)$. Instead, traffic modeling applications require the more accurate estimate given in Definition 2.1. (i). Even if this goes beyond the scope of this work, we observe in Remarks 3.1 and 3.2 that the Riemann solvers detailed in Section 3 below satisfy this property, which would be transmitted to the solutions obtained as limit of wave-front tracking approximations [16].

\section{Riemann problems}

In this section, we detail the construction of the solutions to the two Riemann problems corresponding to the upstream and downstream endpoints of the platoon, located at the flux moving discontinuities $z_{u}(t)$ and $z_{d}(t)$. We therefore consider that these discontinuities move with constant speeds $V_{u}$ and $V_{d}$ and we consider the two interfaces separately.

These Riemann problems provide the necessary information to design a numerical scheme allowing to accurately capture the dynamics at these locations.

\subsection{Front-end of the platoon}

At the downstream endpoint of the platoon, we consider the following Riemann problem:

$$
\begin{cases}\partial_{t} \rho+\partial_{x} F(t, x, \rho)=0 & x \in \mathbb{R}, t>0, \\ \rho(0, x)=\rho_{0}(x)= \begin{cases}\rho_{l} & \text { if } x<z_{d}^{0}, \\ \rho_{r} & \text { if } x \geq z_{d}^{0},\end{cases} \end{cases}
$$

where

$$
F(t, x, \rho)= \begin{cases}f_{\alpha}(\rho) & \text { if } x \leq z_{d}(t) \\ f(\rho) & \text { if } x>z_{d}(t)\end{cases}
$$


with $z_{d}(t):=z_{d}^{0}+V_{d} t, \rho_{l} \in[0, \alpha R], \rho_{r} \in[0, R]$ and under the constraint $V_{d} \leq v\left(\rho_{r}\right)$, to comply with (2.7). (The last condition corresponds to require $\rho_{r} \leq \rho_{d}^{*}$, such that $v\left(\rho_{d}^{*}\right)=V_{d}$.) To solve (3.1), we adapt the concept of supply and demand functions [13, Section 5.2.3] to the platoon reference frame. We denote by $\rho^{\sharp}$ the (unique) solution of the equation $f^{\prime}(\rho)=V_{d}$ and by $\rho_{\alpha}^{\sharp}$ the solution of the equation $f_{\alpha}^{\prime}(\rho)=V_{d}$. Therefore we get

$$
\widetilde{D}^{\alpha}\left(\rho_{l}\right)=\left\{\begin{array}{ll}
f_{\alpha}\left(\rho_{l}\right) & \text { if } \rho_{l}<\rho_{\alpha}^{\sharp}, \\
y_{d}^{\alpha}\left(\rho_{l} ; \rho_{\alpha}^{\sharp}\right) & \text { if } \rho_{l} \geq \rho_{\alpha}^{\sharp},
\end{array} \quad \widetilde{S}\left(\rho_{r}\right)= \begin{cases}y_{d}\left(\rho_{r} ; \rho^{\sharp}\right) & \text { if } \rho_{r}<\rho^{\sharp}, \\
f\left(\rho_{r}\right) & \text { if } \rho_{r} \geq \rho^{\sharp},\end{cases}\right.
$$

where we have denoted

$$
\begin{aligned}
y_{d}^{\alpha}(\rho ; \bar{\rho}) & =f_{\alpha}(\bar{\rho})+V_{d}(\rho-\bar{\rho}), \\
y_{d}(\rho ; \bar{\rho}) & =f(\bar{\rho})+V_{d}(\rho-\bar{\rho}),
\end{aligned}
$$

the straight lines with slope $V_{d}$ passing respectively through $\left(\bar{\rho}, f_{\alpha}(\bar{\rho})\right)$ and $(\bar{\rho}, f(\bar{\rho}))$. Finally, we define for $\bar{\rho} \in[0, \alpha R]$

$$
\begin{aligned}
& \rho^{+}(\bar{\rho}):=\max \left\{\rho \in[0, R]: y_{d}^{\alpha}(\rho ; \bar{\rho})=f(\rho)\right\}, \\
& \rho^{-}(\bar{\rho}):=\min \left\{\rho \in[0, R]: y_{d}^{\alpha}(\rho ; \bar{\rho})=f(\rho)\right\},
\end{aligned}
$$

and for for $\bar{\rho} \in[0, R]$

$$
\begin{aligned}
\rho_{\alpha}^{+}(\bar{\rho}) & :=\max \left\{\rho \in[0, \alpha R]: y_{d}(\rho ; \bar{\rho})=f_{\alpha}(\rho)\right\}, \\
\rho_{\alpha}^{-}(\bar{\rho}) & :=\min \left\{\rho \in[0, \alpha R]: y_{d}(\rho ; \bar{\rho})=f_{\alpha}(\rho)\right\} .
\end{aligned}
$$

We distinguish the following cases.

(D1) $\rho_{l}<\rho_{\alpha}^{\sharp}$ and $\rho_{r}<\rho^{+}\left(\rho_{l}\right)$.

Since in the platoon reference frame we have (with abuse of notation) $\widetilde{D}^{\alpha}\left(\rho_{l}\right)<\widetilde{S}\left(\rho_{r}\right)$, i.e.

$$
y_{d}^{\alpha}\left(\rho ; \rho_{l}\right) \leq \widetilde{S}\left(\rho_{r}\right)+V_{d}\left(\rho-\rho_{r}\right),
$$

the solution consists of a jump discontinuity between $\rho_{l}$ and $\rho^{-}\left(\rho_{l}\right)$, followed by a classical wave (shock or rarefaction) between $\rho^{-}\left(\rho_{l}\right)$ and $\rho_{r}$. In particular, the left and right traces at $x=z_{d}(t)$ are respectively

$$
\hat{\rho}_{\alpha}=\rho_{l}, \quad \check{\rho}=\rho^{-}\left(\rho_{l}\right) .
$$

Figure 1(a) illustrates the solution, the red line representing the jump across the flux discontinuity.

(D2) $\rho_{l}<\rho_{\alpha}^{\sharp}$ and $\rho_{r} \geq \rho^{+}\left(\rho_{l}\right)$.

In this situation computing demand and supply in the platoon reference frame we get $\widetilde{D}^{\alpha}\left(\rho_{l}\right) \geq \widetilde{S}\left(\rho_{r}\right)$. The solution is given by a shock wave between $\rho_{l}$ and $\rho_{\alpha}^{+}\left(\rho_{r}\right)$, followed by a jump discontinuity between $\rho_{\alpha}^{+}\left(\rho_{r}\right)$ and $\rho_{r}$. In this case

$$
\hat{\rho}_{\alpha}=\rho_{\alpha}^{+}\left(\rho_{r}\right), \quad \check{\rho}=\rho_{r},
$$

see also Figure 1(b). 
(D3) $\rho_{l} \geq \rho_{\alpha}^{\sharp}$ and $\rho_{r}<\rho^{+}\left(\rho_{\alpha}^{\sharp}\right)$.

Given these initial data, we have a rarefaction wave between $\rho_{l}$ and $\rho_{\alpha}^{\sharp}$, then a jump between $\rho_{\alpha}^{\sharp}$ and $\rho^{-}\left(\rho_{\alpha}^{\sharp}\right)$ followed by a shock between $\rho^{-}\left(\rho_{\alpha}^{\sharp}\right)$ and $\rho_{r}$, as reported in Figure 1(c). In particular

$$
\hat{\rho}_{\alpha}=\rho_{\alpha}^{\sharp}, \quad \check{\rho}=\rho^{-}\left(\rho_{\alpha}^{\sharp}\right) .
$$

(D4) $\rho_{l} \geq \rho_{\alpha}^{\sharp}$ and $\rho_{r} \geq \rho^{+}\left(\rho_{\alpha}^{\sharp}\right)$.

In this case $\widetilde{D}^{\alpha}\left(\rho_{l}\right) \geq \widetilde{S}\left(\rho_{r}\right)$ and the solution is then given by a (shock or rarefaction) wave between $\rho_{l}$ and $\rho_{\alpha}^{+}\left(\rho_{r}\right)$ and a jump discontinuity between $\rho_{\alpha}^{+}\left(\rho_{r}\right)$ and $\rho_{r}$. We have again

$$
\hat{\rho}_{\alpha}=\rho_{\alpha}^{+}\left(\rho_{r}\right), \quad \check{\rho}=\rho_{r},
$$

see also Figure $1(\mathrm{~d})$,

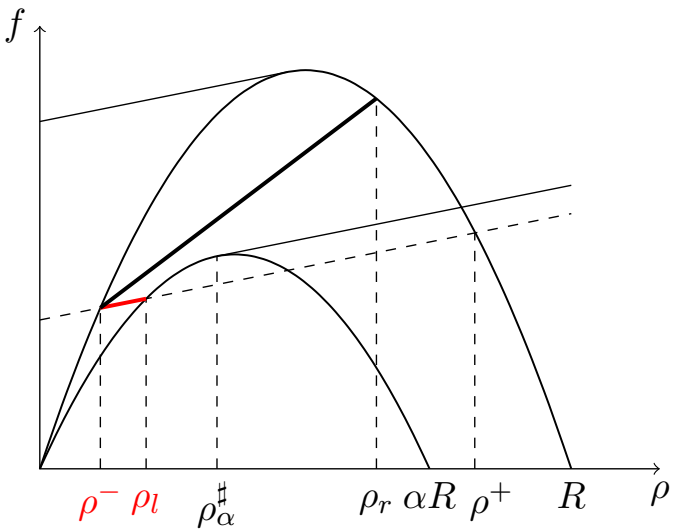

(a) Solution for $\rho_{l}<\rho_{\alpha}^{\sharp}$ and $\rho_{r}<\rho^{+}\left(\rho_{l}\right)$

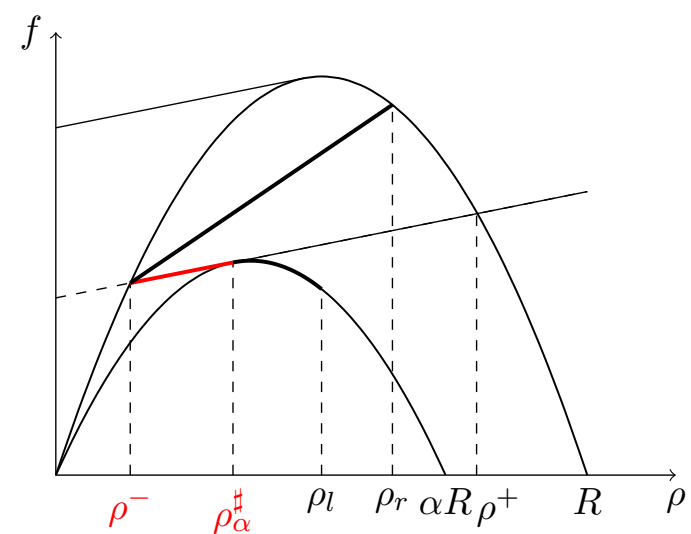

(c) Solution for $\rho_{l} \geq \rho_{\alpha}^{\sharp}$ and $\rho_{r}<\rho^{+}\left(\rho_{\alpha}^{\sharp}\right)$

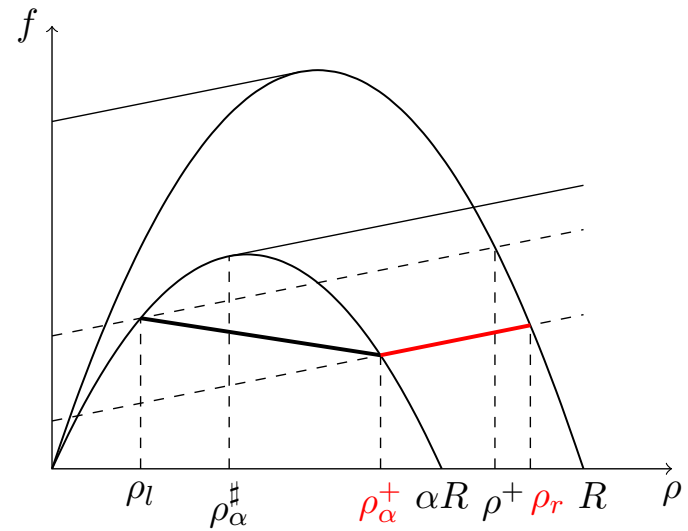

(b) Solution for $\rho_{l}<\rho_{\alpha}^{\sharp}$ and $\rho_{r} \geq \rho^{+}\left(\rho_{l}\right)$

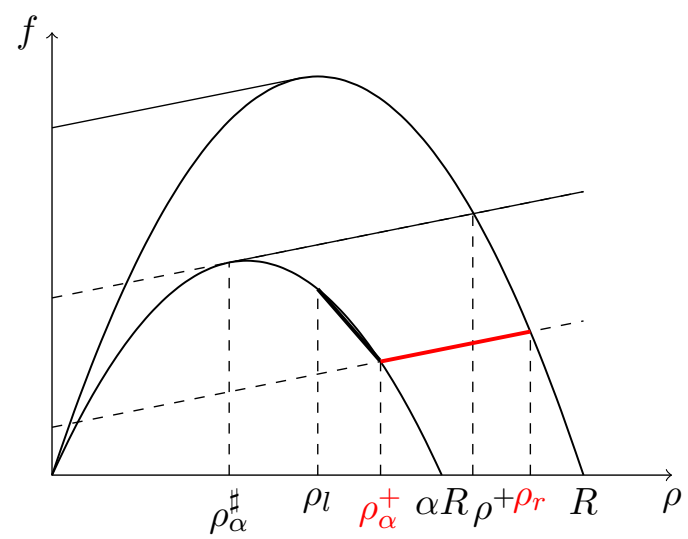

(d) Solution for $\rho_{l} \geq \rho_{\alpha}$ and $\rho_{r} \geq \tilde{\rho}$

Figure 1: Solutions to the Riemann problem (3.1) for different initial data. The non-classical shock corresponding to the flux discontinuity is depicted in red. 
Remark 3.1. The above construction ensures that the solution $\rho(t, x)$ of (3.1) satisfies the maximum principle

$$
\rho(t, x) \in[0, \alpha R] \text { for } x<z_{d}(t) \quad \text { and } \quad \rho(t, x) \in[0, R] \text { for } x>z_{d}(t) .
$$

\subsection{Back-end of the platoon}

At the upstream endpoint of the platoon, the Riemann problem is:

$$
\left\{\begin{array}{l}
\partial_{t} \rho+\partial_{x} F(t, x, \rho)=0 \\
\rho(0, x)=\rho_{0}(x)= \begin{cases}\rho_{l} & \text { if } x<z_{u}^{0}, \\
\rho_{r} & \text { if } x \geq z_{u}^{0},\end{cases}
\end{array}\right.
$$

where

$$
F(t, x, \rho)= \begin{cases}f(\rho) & \text { if } x<z_{u}(t), \\ f_{\alpha}(\rho) & \text { if } x \geq z_{u}(t)\end{cases}
$$

with $z_{u}(t):=z_{u}^{0}+V_{u} t, \rho_{l} \in[0, R]$ and $\rho_{r} \in[0, \alpha R]$. The supply and demand functions become

$$
\widetilde{D}\left(\rho_{l}\right)=\left\{\begin{array}{ll}
f\left(\rho_{l}\right) & \text { if } \rho_{l}<\rho^{\sharp}, \\
y_{u}\left(\rho_{l} ; \rho^{\sharp}\right) & \text { if } \rho_{l} \geq \rho^{\sharp},
\end{array} \quad \widetilde{S}^{\alpha}\left(\rho_{r}\right)= \begin{cases}y_{u}^{\alpha}\left(\rho_{r} ; \rho_{\alpha}^{\sharp}\right) & \text { if } \rho_{r}<\rho_{\alpha}^{\sharp}, \\
f_{\alpha}\left(\rho_{r}\right) & \text { if } \rho_{r} \geq \rho_{\alpha}^{\sharp},\end{cases}\right.
$$

where we have denoted

$$
\begin{aligned}
& y_{u}^{\alpha}(\rho ; \bar{\rho})=f_{\alpha}(\bar{\rho})+V_{u}(\rho-\bar{\rho}), \\
& y_{u}(\rho ; \bar{\rho})=f(\bar{\rho})+V_{u}(\rho-\bar{\rho}) .
\end{aligned}
$$

As in Section 3.1, we define for $\bar{\rho} \in[0, \alpha R]$

$$
\begin{aligned}
& \rho^{+}(\bar{\rho}):=\max \left\{\rho \in[0, R]: y_{u}^{\alpha}(\rho ; \bar{\rho})=f(\rho)\right\}, \\
& \rho^{-}(\bar{\rho}):=\min \left\{\rho \in[0, R]: y_{u}^{\alpha}(\rho ; \bar{\rho})=f(\rho)\right\},
\end{aligned}
$$

and for for $\bar{\rho} \in[0, R]$

$$
\begin{aligned}
& \rho_{\alpha}^{+}(\bar{\rho}):=\max \left\{\rho \in[0, \alpha R]: y_{u}(\rho ; \bar{\rho})=f_{\alpha}(\rho)\right\} \\
& \rho_{\alpha}^{-}(\bar{\rho}):=\min \left\{\rho \in[0, \alpha R]: y_{u}(\rho ; \bar{\rho})=f_{\alpha}(\rho)\right\}
\end{aligned}
$$

We distinguish the following cases.

(U1) $\rho_{l} \leq \rho^{-}\left(\rho_{\alpha}^{\sharp}\right)$ and $\rho_{r} \leq \rho_{\alpha}^{+}\left(\rho_{l}\right)$.

In this situation, in the moving reference frame we get $\widetilde{D}\left(\rho_{l}\right) \leq \widetilde{S}_{\alpha}\left(\rho_{r}\right)$ and the solution consists in a non-classical shock between $\rho_{l}$ and $\rho_{\alpha}^{-}\left(\rho_{l}\right)$, followed by a classical wave (shock or rarefaction) between $\rho_{\alpha}^{-}\left(\rho_{l}\right)$ and $\rho_{r}$, as depicted in Figure 2(a). In this case we define

$$
\hat{\rho}=\rho_{l}, \quad \check{\rho}_{\alpha}=\rho_{\alpha}^{-}\left(\rho_{l}\right) .
$$


(U2) $\rho_{l} \leq \rho^{-}\left(\rho_{\alpha}^{\sharp}\right)$ and $\rho_{r}>\rho_{\alpha}^{+}\left(\rho_{l}\right)$ (this requires $\rho_{\alpha}^{+}\left(\rho_{l}\right)<\alpha R$ if $\left.V_{u}<0\right)$.

Unlike the previous case, now in the moving reference frame it holds $\widetilde{D}\left(\rho_{l}\right)>\widetilde{S}_{\alpha}\left(\rho_{r}\right)$. We then get a classical shock from $\rho_{l}$ to $\rho^{+}\left(\rho_{r}\right)$ and a non-classical shock between $\rho^{+}\left(\rho_{r}\right)$ and $\rho_{r}$, as showed in Figure 2(b). For this, we need $\rho^{+}\left(\rho_{r}\right) \geq R$, thus $V_{u} \geq-f_{\alpha}\left(\rho_{r}\right) /\left(R-\rho_{r}\right)$. We set

$$
\hat{\rho}=\rho^{+}\left(\rho_{r}\right), \quad \check{\rho}_{\alpha}=\rho_{r} .
$$

(U3) $\rho_{l}>\rho^{-}\left(\rho_{\alpha}^{\sharp}\right)$ and $\rho_{r} \leq \rho_{\alpha}^{\sharp}$.

In the moving reference frame, $\widetilde{D}\left(\rho_{l}\right)>\widetilde{S}_{\alpha}\left(\rho_{r}\right)$, and the solution, depicted in Figure 2(c), is given by a classical wave between $\rho_{l}$ and $\rho^{+}\left(\rho_{\alpha}^{\sharp}\right)$, followed by a non-classical jump joining $\rho^{+}\left(\rho_{\alpha}^{\sharp}\right)$ to $\rho_{\alpha}^{\sharp}$ and a rarefaction wave from $\rho_{\alpha}^{\sharp}$ to $\rho_{r}$. For this, we need $\rho^{+}\left(\rho_{\alpha}^{\sharp}\right) \geq R$, thus $V_{u} \geq-f_{\alpha}\left(\rho_{\alpha}^{\sharp}\right) /\left(R-\rho_{\alpha}^{\sharp}\right)$. This condition is weaker than the previous one. Therefore,

$$
\hat{\rho}=\rho^{+}\left(\rho_{\alpha}^{\sharp}\right), \quad \check{\rho}_{\alpha}=\rho_{\alpha}^{\sharp} .
$$

(U4) $\rho_{l}>\rho^{-}\left(\rho_{\alpha}^{\sharp}\right)$ and $\rho_{r}>\rho_{\alpha}^{\sharp}$.

In the moving reference frame $\widetilde{D}\left(\rho_{l}\right)>\widetilde{S}_{\alpha}\left(\rho_{r}\right)$ and we get a classical wave (shock or rarefaction) between $\rho_{l}$ and $\rho^{+}\left(\rho_{r}\right)$, followed by non-classical discontinuity between $\rho^{+}\left(\rho_{r}\right)$ and $\rho_{r}$, see Figure $2(\mathrm{~d})$. Also here, we need $\rho^{+}\left(\rho_{r}\right) \geq R$, thus $V_{u} \geq-f_{\alpha}\left(\rho_{r}\right) /(R-$ $\left.\rho_{r}\right)$. We set

$$
\hat{\rho}=\rho^{+}\left(\rho_{r}\right), \quad \check{\rho}_{\alpha}=\rho_{r} .
$$

Remark 3.2. The above construction ensures that the solution $\rho(t, x)$ of (3.2) satisfies the maximum principle

$$
\rho(t, x) \in[0, R] \text { for } x<z_{u}(t) \quad \text { and } \quad \rho(t, x) \in[0, \alpha R] \text { for } x>z_{u}(t) .
$$

\section{Numerical scheme}

To approximate the solution of the conservation law (2.1a), we design a conservative finite volume scheme based on flux discontinuities reconstruction. Given the fixed space and time steps $\Delta x$ and $\Delta t$, we denote $x_{j}=(j-1 / 2) \Delta x$ the cell centers, $x_{j+1 / 2}=j \Delta x$ the cell interfaces, for $j \in \mathbb{Z}$, and $t^{n}=n \Delta t, n \in \mathbb{N}$, the time mesh.

We approximate the initial datum (2.1b) by the piece-wise constant function given by its average on each discretization cell $C_{j}=\left[x_{j-1 / 2}, x_{j+1 / 2}[\right.$, i.e.

$$
\rho_{j}^{0}=\frac{1}{\Delta x} \int_{x_{j-1 / 2}}^{x_{j+1 / 2}} \rho_{0}(x) d x, \quad j \in \mathbb{Z} .
$$

At each time step $n$ the density is updated as

$$
\rho_{j}^{n+1}=\rho_{j}^{n}-\frac{\Delta t}{\Delta x}\left(F_{j+1 / 2}^{n}-F_{j-1 / 2}^{n}\right), \quad j \in \mathbb{Z},
$$

with $2 V \Delta t \leq \Delta x$, where the numerical flux $F_{j+1 / 2}^{n}$ is given by the standard Godunov's flux [14] away from the (approximate) space discontinuities locations $z_{u}^{n}$ and $z_{d}^{n}$. For concave flux functions as (2.3) $-(2.4)$, this is equivalent to the supply-demand method (or Cell Transmission 


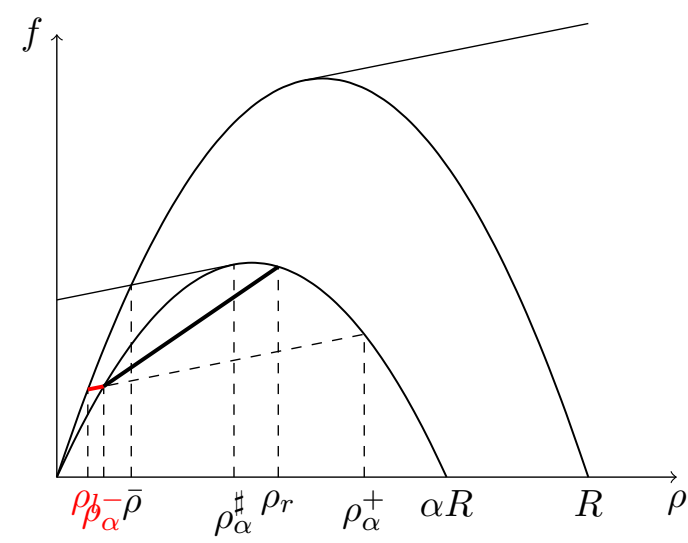

(a) Solution for $\rho_{l} \leq \rho^{-}\left(\rho_{\alpha}^{\sharp}\right)$ and $\rho_{r} \leq \rho_{\alpha}^{+}\left(\rho_{l}\right)$

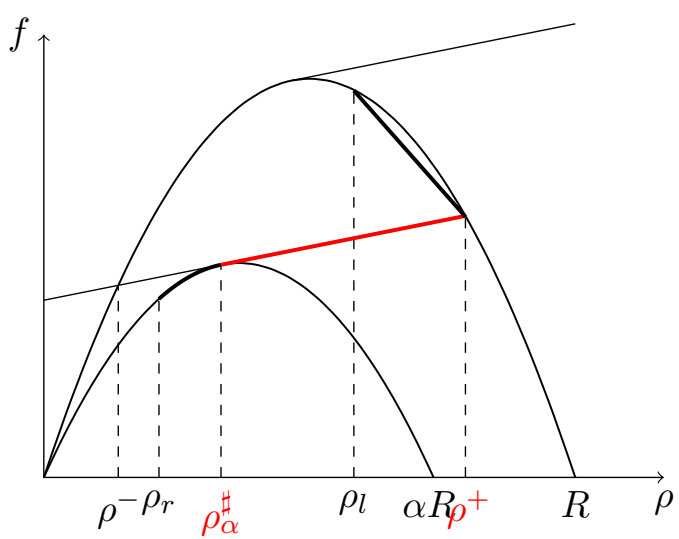

(c) Solution for $\rho_{l}>\rho^{-}\left(\rho_{\alpha}^{\sharp}\right)$ and $\rho_{r} \leq \rho_{\text {alpha }}^{\sharp}$

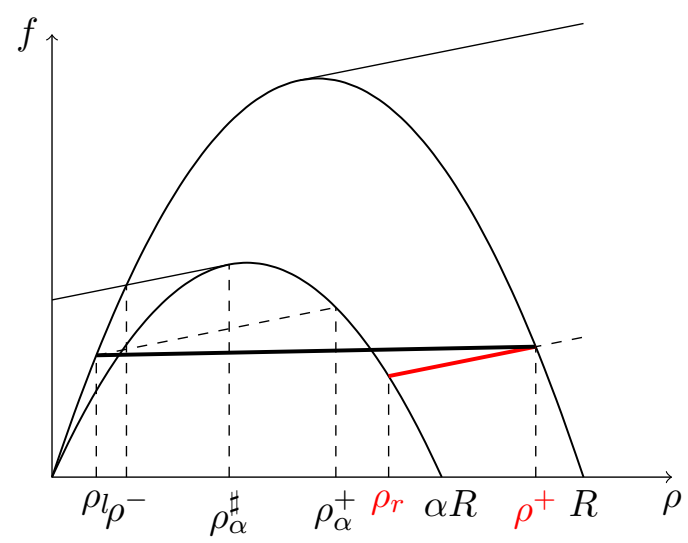

(b) Solution for $\rho_{l} \leq \rho^{-}\left(\rho_{\alpha}^{\sharp}\right)$ and $\rho_{r}>\rho_{\alpha}^{+}\left(\rho_{l}\right)$

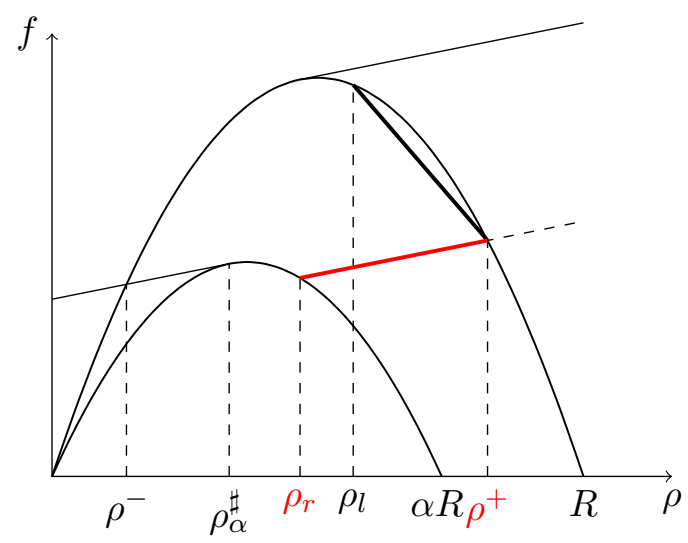

(d) Solution for $\rho_{l}>\rho^{-}\left(\rho_{\alpha}^{\sharp}\right)$ and $\rho_{r}>\rho_{\alpha}^{\sharp}$

Figure 2: Solutions to the Riemann problem (3.2) for different initial data. The non-classical shock corresponding to the flux discontinuity is depicted in red.

Model) used by the transportation engineers [9, 13, 20]. More precisely, if $z_{u}^{n} \in C_{j_{u}}$ and $z_{d}^{n} \in C_{j_{d}}$ for some indexes $j_{u}, j_{d} \in \mathbb{Z}$, the demand and the supply functions are defined as

$$
D\left(\rho_{j}^{n}\right)=\left\{\begin{array}{ll}
f\left(\rho_{j}^{n}\right) & \text { if } \rho_{j}^{n}<\rho_{c r}, \\
f^{\max } & \text { if } \rho_{j}^{n} \geq \rho_{c r},
\end{array} \quad S\left(\rho_{j}^{n}\right)= \begin{cases}f^{\max } & \text { if } \rho_{j}^{n}<\rho_{c r}, \\
f\left(\rho_{j}^{n}\right) & \text { if } \rho_{j}^{n} \geq \rho_{c r},\end{cases}\right.
$$

for $j<j_{u}$ or $j>j_{d}$. Similarly, for $j_{u}<j<j_{d}$, the demand and supply are computed considering the reduced flow:

$$
D^{\alpha}\left(\rho_{j}^{n}\right)=\left\{\begin{array}{ll}
f_{\alpha}\left(\rho_{j}^{n}\right) & \text { if } \rho_{j}^{n}<\rho_{c r}^{\alpha}, \\
f_{\alpha}^{\max } & \text { if } \rho_{j}^{n} \geq \rho_{c r}^{\alpha},
\end{array} \quad S^{\alpha}\left(\rho_{j}^{n}\right)= \begin{cases}f_{\alpha}^{\max } & \text { if } \rho_{j}^{n}<\rho_{c r}^{\alpha}, \\
f_{\alpha}\left(\rho_{j}^{n}\right) & \text { if } \rho_{j}^{n} \geq \rho_{c r}^{\alpha} .\end{cases}\right.
$$

See Figure 3 ,

Therefore, the numerical fluxes $F_{j+1 / 2}^{n}$ are simply defined as

$$
\begin{aligned}
& F_{j+1 / 2}^{n}=\min \left\{D\left(\rho_{j-1}^{n}\right), S\left(\rho_{j}^{n}\right)\right\} \quad \text { for } j \leq j_{u}-2 \text { and } j \geq j_{d}+1 \text {, } \\
& F_{j+1 / 2}^{n}=\min \left\{D^{\alpha}\left(\rho_{j-1}^{n}\right), S^{\alpha}\left(\rho_{j}^{n}\right)\right\} \quad \text { for } j \geq j_{u}+1 \text { and } j \leq j_{d}-2 \text {, }
\end{aligned}
$$



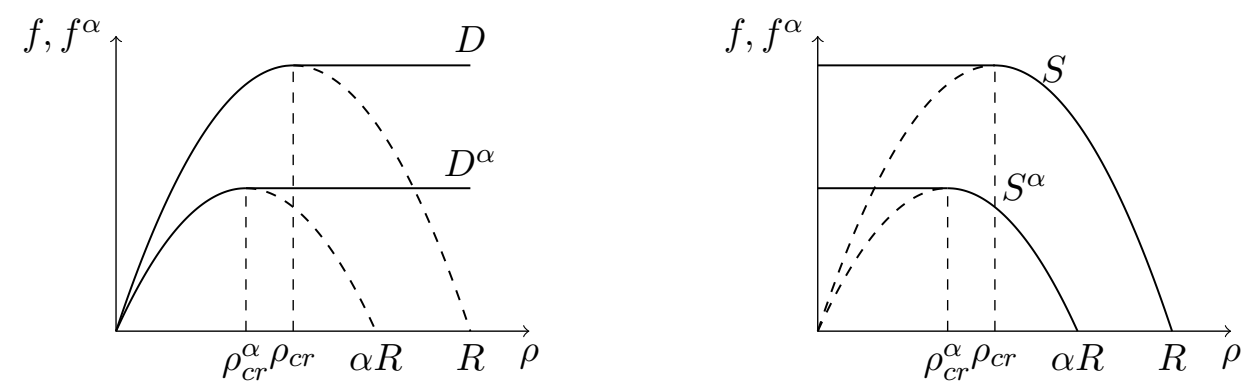

Figure 3: Demand and supply considering the normal and rescaled flow

where we are assuming, without loss of generality, that $j_{u} \leq j_{d}-3$. The interfaces $x_{j_{u} \pm 1 / 2}$ and $x_{j_{d} \pm 1 / 2}$ need a special treatment due to the presence of the flux discontinuities. We will describe the procedure separately for the two cases below.

Besides, the position of the downstream endpoint of the platoon is updated as

$$
z_{d}^{n+1}=z_{d}^{n}+\min \left\{V_{d}^{n}, v\left(\rho_{j_{d}+1}^{n}\right)\right\} \Delta t,
$$

while the position of the upstream endpoint of the platoon is updated considering its own speed $V_{u}$.

$$
z_{u}^{n+1}=z_{u}^{n}+V_{u}^{n} \Delta t
$$

where we have set $V_{u, d}^{n}:=\frac{1}{\Delta t} \int_{t^{n}}^{t^{n+1}} V_{u, d}(t) d t$.

Remark 4.1. The trajectory of the downstream endpoint can be approximated more carefully following [6, Section 3.2], see also [5].

\subsection{Front-end of the platoon}

We expect a discontinuity to appear in cell $C_{j_{d}}$ due to the presence of the flux discontinuity induced by the platoon downstream endpoint. This corresponds to the Riemann problem (3.1), where we set $\rho_{l}=\rho_{j_{d}-1}^{n}$ and $\rho_{r}=\rho_{j_{d}+1}^{n}$. Following [3, 6], the idea is to replace the density $\rho_{j_{d}}^{n}$ in the cell $C_{j_{d}}$ by a convex combination of the values $\hat{\rho}_{\alpha}$ and $\check{\rho}$ corresponding to $\rho_{l}$ and $\rho_{r}$, as computed in Section 3.1. More precisely, we compute $d_{j_{d}}$ such that

$$
\hat{\rho}_{\alpha} d_{j_{d}}+\check{\rho}\left(1-d_{j_{d}}\right)=\rho_{j_{d}}^{n}, \quad \text { i.e. } \quad d_{j_{d}}=\frac{\rho_{j_{d}}^{n}-\check{\rho}}{\hat{\rho}_{\alpha}-\check{\rho}} .
$$

The reconstructed discontinuity is then located at $\bar{x}_{j_{d}}=x_{j_{d}-1 / 2}+d_{j_{d}} \Delta x$, guaranteeing mass conservation.

If $d_{j_{d}} \in[0,1]$, the numerical flux at the interface $x_{j_{d}+1 / 2}$ is reconstructed as

$$
\Delta t F_{j_{d}+1 / 2}^{n}=\min \left\{\Delta t_{j_{d}}, \Delta t\right\} f(\check{\rho})+\max \left\{\Delta t-\Delta t_{j_{d}}, 0\right\} f_{\alpha}\left(\hat{\rho}_{\alpha}\right)
$$

where

$$
\Delta t_{j_{d}}=\frac{1-d_{j_{d}}}{\min \left\{V_{d}^{n}, v\left(\rho_{j_{d}+1}^{n}\right)\right\}} \Delta x
$$

is the time needed by the discontinuity traveling at speed $v_{d}$ to reach the interface $x_{j_{d}+1 / 2}$. 


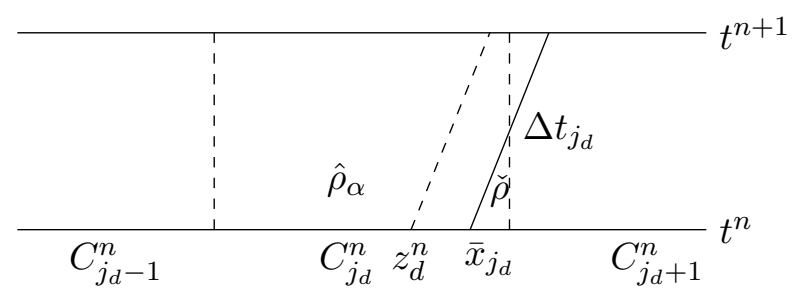

Figure 4: Representation of the reconstruction algorithm (4.1)-(4.2) at the downstream endpoint of the platoon.

The numerical flux at $x=x_{j_{d}-1 / 2}$ is computed as

$$
F_{j_{d}-1 / 2}^{n}=\min \left\{D^{\alpha}\left(\rho_{j_{d}-1}^{n}\right), S^{\alpha}\left(\hat{\rho}_{\alpha}\right)\right\} .
$$

The idea of the above scheme is represented in Figure 4,

If $\rho_{j_{d}}^{n} \notin\left[\min \left\{\hat{\rho}_{\alpha}, \check{\rho}\right\}, \max \left\{\hat{\rho}_{\alpha}, \check{\rho}\right\}\right]$, we get $d_{j_{d}} \notin[0,1]$. For example, due to the approximation introduced considering $\rho_{l}=\rho_{j_{d}-1}^{n}$ and $\rho_{r}=\rho_{j_{d}+1}^{n}$, the discontinuity $\bar{x}_{j_{d}}=x_{j_{d}-1 / 2}+d_{j_{d}} \Delta x$ does not coincide in general with $z_{d}^{n}$, the actual position of the front of the platoon, even if they travel at the same speed. In particular, it may happen that $z_{d}^{n}$ and $\bar{x}_{j_{d}}$ are not located in the same cell, as depicted in Fig,4,

In this situation, we adopt the following strategy:

- if $\left|\rho_{j_{d}}^{n}-\hat{\rho}_{\alpha}\right|<\left|\rho_{j_{d}}^{n}-\check{\rho}\right|$, i.e. $d_{j_{d}}>1$, we set $\hat{\rho}_{\alpha}^{\prime}=\rho_{j_{d}}^{n}$ and $\check{\rho}^{\prime}=\check{\rho}$, which corresponds to $d_{j_{d}}^{\prime}=1$; the corresponding numerical fluxes are then computed as

$$
\begin{aligned}
& F_{j_{d}-1 / 2}^{n}=\min \left\{D^{\alpha}\left(\rho_{j_{d}-1}^{n}\right), S^{\alpha}\left(\rho_{j_{d}}^{n}\right)\right\} \\
& F_{j_{d}+1 / 2}^{n}=\min \left\{D^{\alpha}\left(\rho_{j_{d}}^{n}\right), S\left(\rho_{j_{d}+1}^{n}\right)\right\}
\end{aligned}
$$

- if $\left|\rho_{j_{d}}^{n}-\hat{\rho}_{\alpha}\right|>\left|\rho_{j_{d}}^{n}-\check{\rho}\right|$, i.e. $d_{j_{d}}<0$, we take $\hat{\rho}_{\alpha}^{\prime}=\hat{\rho}_{\alpha}$ and $\check{\rho}^{\prime}=\rho_{j_{d}}^{n}$; the numerical fluxes are then computed as

$$
\begin{aligned}
& F_{j_{d}-1 / 2}^{n}=\min \left\{D^{\alpha}\left(\rho_{j_{d}-1}^{n}\right), S^{\alpha}\left(\hat{\rho}_{\alpha}\right)\right\} \\
& F_{j_{d}+1 / 2}^{n}=\min \left\{D\left(\rho_{j_{d}}^{n}\right), S\left(\rho_{j_{d}+1}^{n}\right)\right\}
\end{aligned}
$$

which takes into account the direction of propagation of the non-classical discontinuity and corresponds to (4.1)-(4.2) with $\hat{\rho}_{\alpha}^{\prime}, \check{\rho}^{\prime}$ in place of $\hat{\rho}_{\alpha}, \check{\rho}$.

\subsection{Back-end of the platoon}

We apply the same approach adopted for the front of the platoon to the discontinuity arising at the upstream endpoint of the platoon in the cell $C_{j_{u}}$, corresponding to the Riemann problem (3.2). We set $\rho_{l}=\rho_{j_{u}-1}^{n}$ and $\rho_{r}=\rho_{j_{u}+1}^{n}$ and we compute

$$
d_{j_{u}}=\frac{\rho_{j_{u}}^{n}-\check{\rho}_{\alpha}}{\hat{\rho}-\check{\rho}_{\alpha}}
$$




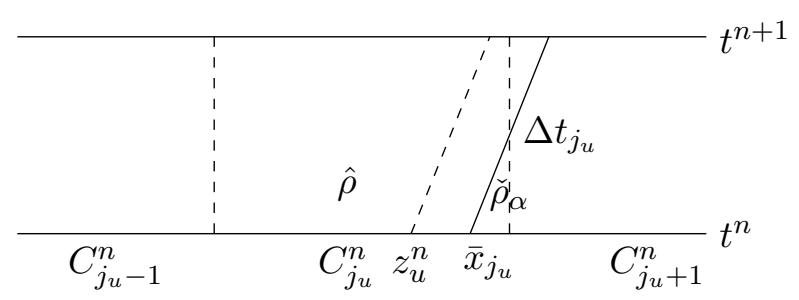

(a) Case $V_{u}^{n} \geq 0$.

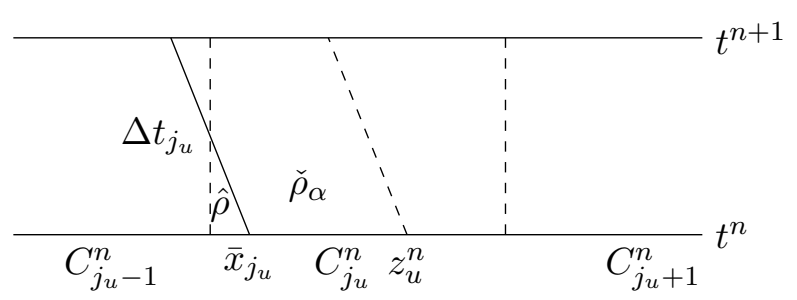

(b) Case $V_{u}^{n}<0$.

Figure 5: Representation of the reconstruction algorithm at the upstream endpoint of the platoon.

where $\hat{\rho}, \check{\rho}_{\alpha}$ are given in Section 3.2 .

If $d_{j_{u}} \in[0,1]$ and $V_{u}^{n} \geq 0$, the numerical flux at the interface $x_{j_{u}+1 / 2}$ is reconstructed as

$$
\Delta t F_{j_{u}+1 / 2}^{n}=\min \left\{\Delta t_{j_{u}}, \Delta t\right\} f_{\alpha}\left(\check{\rho}_{\alpha}\right)+\max \left\{\Delta t-\Delta t_{j_{u}}, 0\right\} f(\hat{\rho}),
$$

where

$$
\Delta t_{j_{u}}=\frac{1-d_{j_{u}}}{V_{u}^{n}} \Delta x
$$

and

see Figure $5(\mathrm{a})$.

$$
F_{j_{u}-1 / 2}^{n}=\min \left\{D\left(\rho_{j_{u}-1}^{n}\right), S(\hat{\rho})\right\}
$$

Instead, if $V_{u}^{n}<0$, we need to reconstruct the flux at the interface $x=x_{j_{u}-1 / 2}$ : we define

$$
\Delta t F_{j_{u}-1 / 2}^{n}=\min \left\{\Delta t_{j_{u}}, \Delta t\right\} f(\hat{\rho})+\max \left\{\Delta t-\Delta t_{j_{u}}, 0\right\} f_{\alpha}\left(\check{\rho}_{\alpha}\right),
$$

where

$$
\Delta t_{j_{u}}=-\frac{d_{j_{u}}}{V_{u}^{n}} \Delta x
$$

is the time for the discontinuity to reach the interface $x_{j_{u}-1 / 2}$. Moreover, we set

$$
F_{j_{u}+1 / 2}^{n}=\min \left\{D^{\alpha}\left(\check{\rho}_{\alpha}\right), S^{\alpha}\left(\rho_{j_{u}+1}^{n}\right)\right\}
$$

see $(5(\mathrm{~b}))$.

In analogy with Section 4.2 , when $d_{j_{u}} \notin[0,1]$, we adapt the strategy as follows:

- if $\left|\rho_{j_{u}}^{n}-\hat{\rho}\right|<\left|\rho_{j_{u}}^{n}-\check{\rho}_{\alpha}\right|$, i.e. $d_{j_{u}}>1$, we set $\hat{\rho}^{\prime}=\rho_{j_{u}}^{n}$ and $\check{\rho}_{\alpha}^{\prime}=\check{\rho}_{\alpha}$ and the corresponding numerical fluxes are then computed as

$$
F_{j_{u}-1 / 2}^{n}=\min \left\{D\left(\rho_{j_{u}-1}^{n}\right), S\left(\rho_{j_{u}}^{n}\right)\right\}
$$




$$
F_{j_{u}+1 / 2}^{n}=\min \left\{D\left(\rho_{j_{u}}^{n}\right), S^{\alpha}\left(\rho_{j_{u}+1}^{n}\right)\right\}
$$

- if $\left|\rho_{j_{u}}^{n}-\hat{\rho}\right|>\left|\rho_{j_{u}}^{n}-\check{\rho}_{\alpha}\right|$, i.e. $d_{j_{u}}<0$, we take $\hat{\rho}^{\prime}=\hat{\rho}$ and $\check{\rho}_{\alpha}^{\prime}=\rho_{j_{u}}^{n}$; the numerical fluxes are then computed as

$$
\begin{aligned}
& F_{j_{u}-1 / 2}^{n}=\min \left\{D\left(\rho_{j_{u}-1}^{n}\right), S(\hat{\rho})\right\} \\
& F_{j_{u}+1 / 2}^{n}=\min \left\{D^{\alpha}\left(\rho_{j_{u}}^{n}\right), S^{\alpha}\left(\rho_{j_{u}+1}^{n}\right)\right\} .
\end{aligned}
$$

\section{$5 \quad$ Numerical simulations}

In this section we report some numerical simulations of the previously described model. The front-end and the back-end of the platoon will be first simulated separately. In the simulation the space is discretized with $\Delta x=0.001$.

\subsection{Front-end of the platoon}

In the following, we set $V=1, R=1, \alpha=0.5, V_{d}=0.3$, which gives $\rho_{\alpha}^{\sharp}=0.1750$.

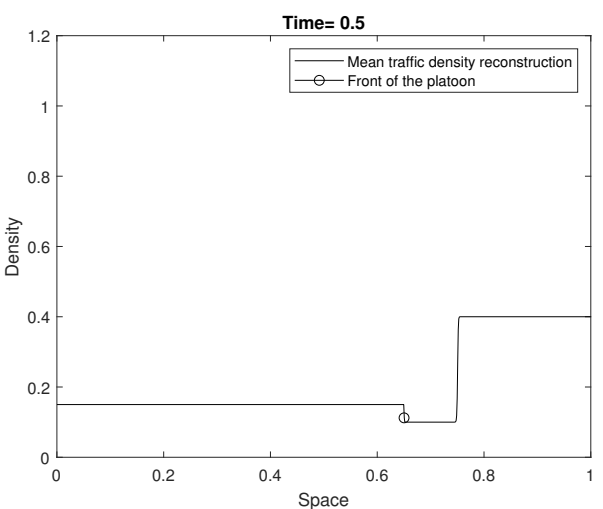

(a) case $\mathrm{d} 1$

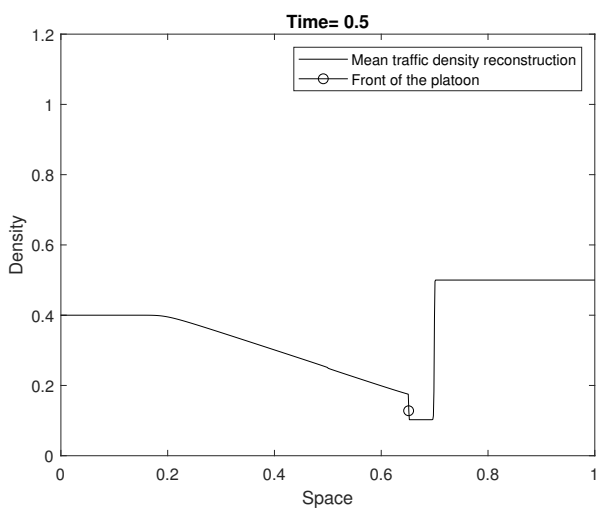

(c) case $\mathrm{d} 3$

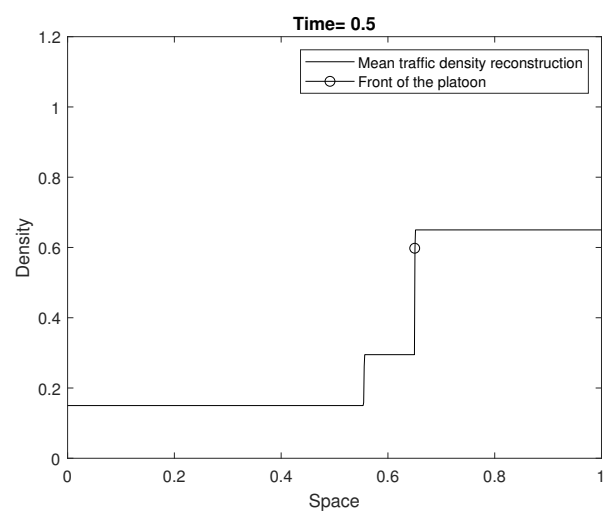

(b) case $\mathrm{d} 2$

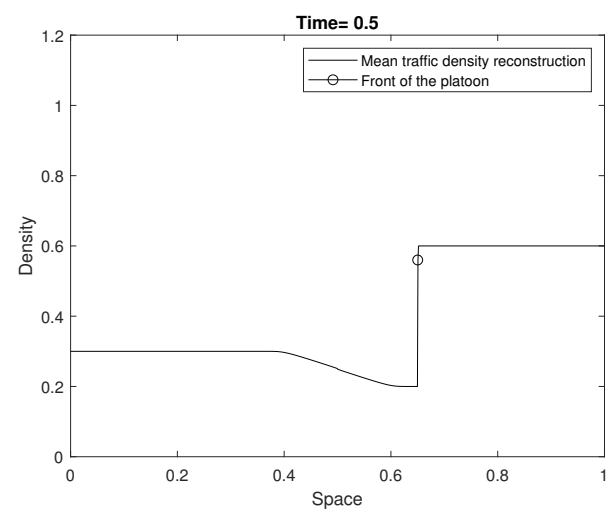

(d) case $\mathrm{d} 4$

Figure 6: Density profile at the front of the platoon at time $t=0.5$ for different initial data. 
Case d1) We consider the Riemann type initial datum

$$
\rho_{0}(x)= \begin{cases}0.15 & \text { if } x<0.5 \\ 0.4 & \text { if } x \geq 0.5\end{cases}
$$

Computing $\hat{\rho}_{\alpha}$ and $\check{\rho}$ as in Section 3.1. (D1), we get $\hat{\rho}_{\alpha}=\rho_{l}=0.15$ and $\check{\rho}=0.1$. In Figure 6(a) we can observe the non-classical shock followed by a classical one, which form the solution.

Case d2) We consider a Riemann type initial datum

$$
\rho_{0}(x)= \begin{cases}0.15 & \text { if } x<0.5 \\ 0.65 & \text { if } x \geq 0.5\end{cases}
$$

This gives $\check{\rho}=0.65$ and $\hat{\rho}_{\alpha}=\rho_{l}=0.29$. The profile of the solution given a classical shock followed by a non-classical one is depicted in Figure 6(b), see also Section 3.1, (D2).

Case d3) We now consider an initial density as

$$
\rho_{0}(x)= \begin{cases}0.4 & \text { if } x<0.5 \\ 0.5 & \text { if } x \geq 0.5\end{cases}
$$

Figure 6(c) illustrate the trend of the solution in this situation. According to Section 3.1. (D3), we compute $\check{\rho}=0.1025$ and $\hat{\rho}_{\alpha}=\rho_{\alpha}^{\sharp}=0.1750$ and we get a rarefaction wave between $\rho_{l}$ and $\hat{\rho}_{\alpha}$, followed by a non-classical and a classical shock.

Case d4) We now consider the initial density

$$
\rho_{0}(x)= \begin{cases}0.3 & \text { if } x<0.5 \\ 0.6 & \text { if } x \geq 0.5\end{cases}
$$

With this initial datum, we obtain a rarefaction wave between $\rho_{l}$ and $\hat{\rho}=0.2$ and then a nonclassical shock to $\check{\rho}=\rho_{r}$ (see Section 3.1, (D4)), as shown in the simulation of Figure 6(d).

\subsection{Back-end of the platoon}

In the following, we take $V=1, R=1, \alpha=0.5, V_{u}=0.2$, corresponding to $\rho_{\alpha}^{\sharp}=0.2$.

Case u1) We consider the Riemann type initial datum

$$
\rho_{0}(x)= \begin{cases}0.08 & \text { if } x<0.5 \\ 0.2 & \text { if } x \geq 0.5\end{cases}
$$

Then $\hat{\rho}=\rho_{l}=0.08$ and $\check{\rho}_{\alpha}=0.0942$, as explained in Section 3.2. (U1). We can observe the non-classical shock followed by the classical one in Figure 7(a).

Case u2) We consider the Riemann type initial datum

$$
\rho_{0}(x)= \begin{cases}0.08 & \text { if } x<0.5 \\ 0.4 & \text { if } x \geq 0.5\end{cases}
$$

According to Section 3.2, (U2), we get $\hat{\rho}=0.8$ and $\check{\rho}_{\alpha}=\rho_{r}=0.4$, and the solution is reported in Figure $7(\mathrm{~b})$. 


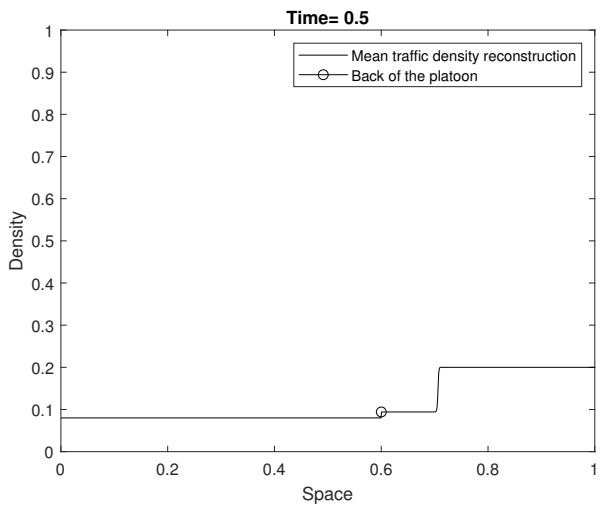

(a) case $u 1$

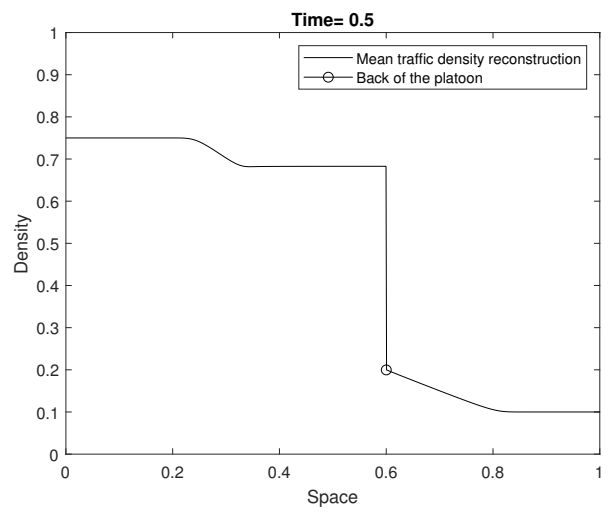

(c) case u3

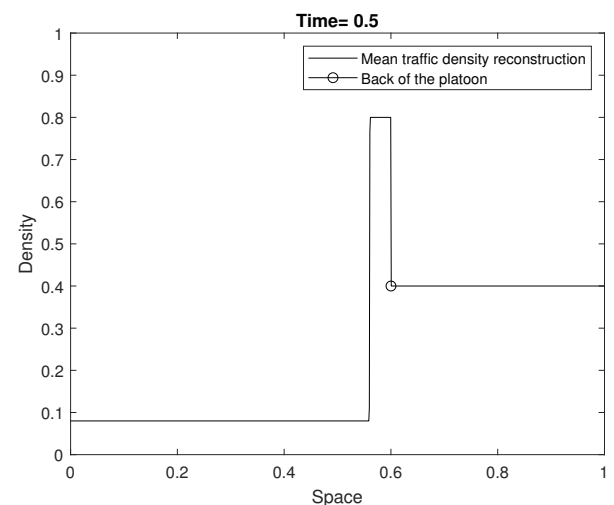

(b) case $u 2$

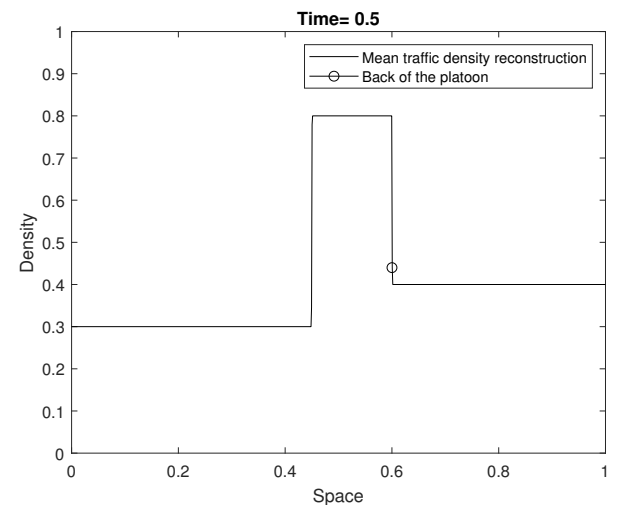

(d) case $\mathrm{u} 4$

Figure 7: Density profile at the back-end of the platoon at time $t=0.5$ for different initial data.

Case u3) We now consider as initial datum the case described in Section 3.2, (U3):

$$
\rho_{0}(x)= \begin{cases}0.75 & \text { if } x<0.5 \\ 0.1 & \text { if } x \geq 0.5\end{cases}
$$

The solution is depicted in Figure 7(c). We have a rarefaction wave between the value $\rho_{l}$ and $\hat{\rho}=0.6828$, followed by the non-classical shock between $\hat{\rho}$ and $\check{\rho}_{\alpha}=\rho_{\alpha}^{\sharp}$, and a rarefaction wave to $\rho_{r}$.

\section{Case u4)}

An initial datum as in Section [3.2, (U4), is here given:

$$
\rho_{0}(x)= \begin{cases}0.3 & \text { if } x<0.5 \\ 0.4 & \text { if } x \geq 0.5\end{cases}
$$

As depicted in Figure 7(d), the solution presents a shock wave between $\rho_{l}$ and $\hat{\rho}=0.8$, followed by the non-classical shock to $\check{\rho}_{\alpha}=\rho_{r}$. 


\subsection{The complete platoon}

Let us now consider the whole model, with the initial and ending points of the platoon. As an example, we consider the following initial datum:

$$
\rho_{0}(x)= \begin{cases}0.3 & \text { if } x<0.2 \\ 0.4 & \text { if } 0.2 \leq x<0.5 \\ 0.5 & \text { if } x \geq 0.5\end{cases}
$$

The initial length of the platoon is $L=0.3$, the initial point of the platoon moves with speed $V_{d}=0.3$ while the ending point has a lower speed $V_{u}=0.2$, thus the platoon will change its length during the simulation. For the final point of the platoon we are in the situation described in Section 3.2. (U4), see also Case u4) in Section 5.2, and we expect a shock to appear with a value of the density equal to the computed $\hat{\rho}=0.8$. For the downstream endpoint of the platoon, we are the situation of Section 3.1. (D3), in which a rarefaction wave followed by a shock appears. The simulation results are reported in Figure 8, 9,

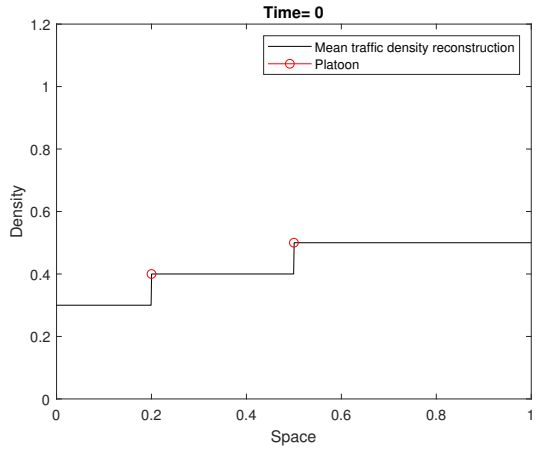

(a)

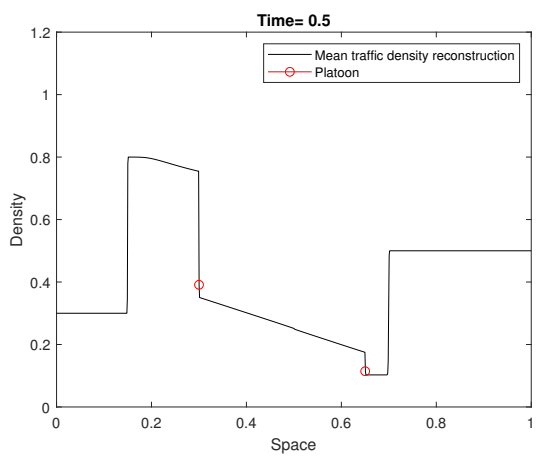

(c)

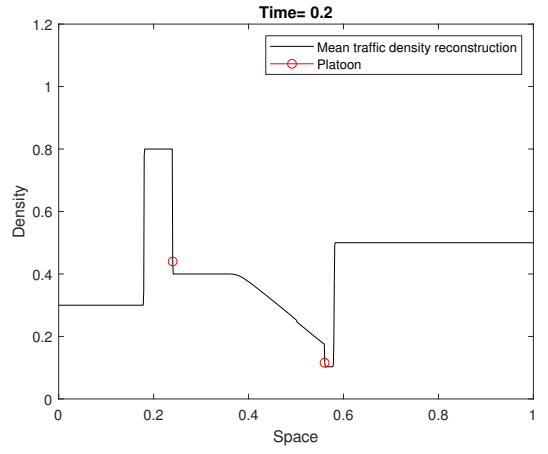

(b)

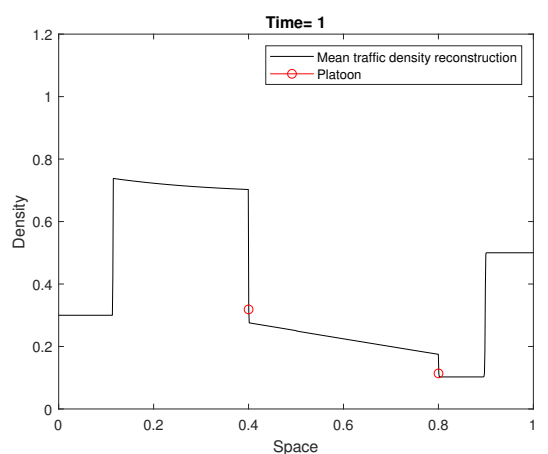

(d)

Figure 8: Density profile at different times corresponding to the initial condition (5.1).

\section{Conclusion}

In this paper, we introduced a model describing the dynamics of length varying platoons in traffic flow by means of a coupled PDE- ODE system with moving constraints. The flow 


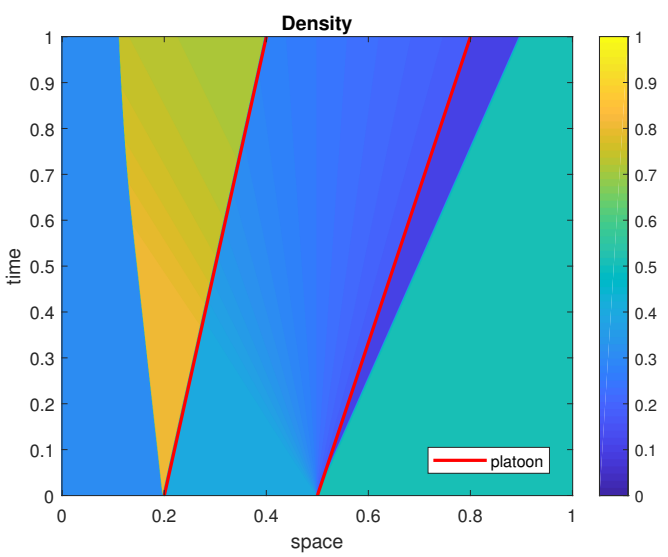

(a) Absolute density

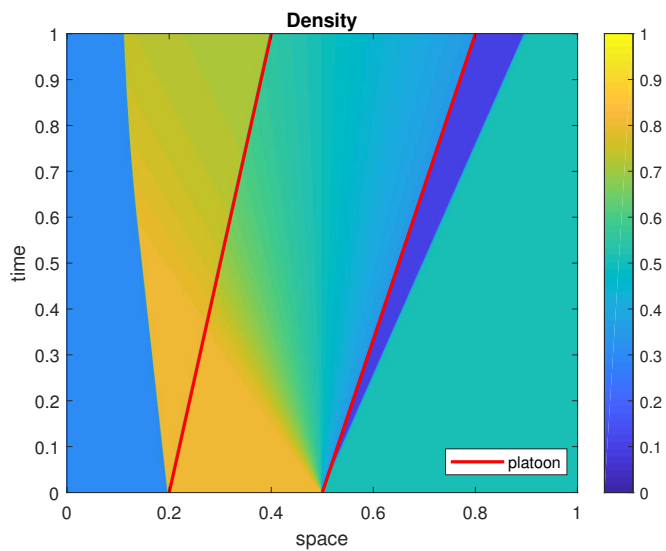

(b) Relative density

Figure 9: Space-time evolution of the solution to (2.1) corresponding to the initial datum (5.1): plot (a) displays the absolute density values $\rho(t, x)$ everywhere, plot (b) accounts for the relative density $\rho(t, x) / \alpha R$ at the platoon location.

discontinuities corresponding to the initial and final points of the platoon are separately studied and a finite volume numerical scheme sharply capturing the solution discontinuities is proposed. Several test cases illustrate the effectiveness of the approach, showing that nonclassical discontinuities arising at platoon endpoints are sharply captured. As future work, the speed and the length of the platoon will be taken as control variables and an optimal control approach will be applied in order to mitigate congestion on the highway.

Acknowledgements: This research was partially accomplished during the first author's visit at Inria Sophia Antipolis - Méditerranée.

The authors are grateful to Christophe Chalons for illuminating discussions.

\section{References}

[1] B. Andreianov, K. H. Karlsen, and N. H. Risebro. A theory of $L^{1}$-dissipative solvers for scalar conservation laws with discontinuous flux. Arch. Ration. Mech. Anal., 201(1):27-86, 2011.

[2] A. K. Bhoopalam, N. Agatz, and R. Zuidwijk. Planning of truck platoons: A literature review and directions for future research. Transportation Research Part B: Methodological, 107:212-228, 2018.

[3] B. Boutin, C. Chalons, F. Lagoutière, and P. G. LeFloch. Convergent and conservative schemes for nonclassical solutions based on kinetic relations. I. Interfaces Free Bound., 10(3):399-421, 2008.

[4] A. Bressan, G. Guerra, and W. Shen. Vanishing viscosity solutions for conservation laws with regulated flux. J. Differential Equations, 266(1):312-351, 2019.

[5] G. Bretti and B. Piccoli. A tracking algorithm for car paths on road networks. SIAM J. Appl. Dyn. Syst., 7(2):510-531, 2008.

[6] C. Chalons, M. L. Delle Monache, and P. Goatin. A conservative scheme for non-classical solutions to a strongly coupled PDE-ODE problem. Interfaces Free Bound., 19(4):553-570, 2017. 
[7] M. Cicic and K. H. Johansson. Traffic regulation via individually controlled automated vehicles: a cell transmission model approach. In Proceedings of the 21st International Conference on Intelligent Transportation Systems (ITSC), pages 766-771. IEEE, nov 2018.

[8] G. M. Coclite and N. H. Risebro. Conservation laws with time dependent discontinuous coefficients. SIAM J. Math. Anal., 36(4):1293-1309, 2005.

[9] C. F. Daganzo. The cell transmission model: A dynamic representation of highway traffic consistent with the hydrodynamic theory. Transportation Research Part B: Methodological, 28(4):269287,1994 .

[10] M. L. Delle Monache and P. Goatin. Scalar conservation laws with moving constraints arising in traffic flow modeling: an existence result. J. Differential Equations, 257(11):4015-4029, 2014.

[11] N. S. Dymski, P. Goatin, and M. D. Rosini. Existence of BV solutions for a non-conservative constrained Aw-Rascle-Zhang model for vehicular traffic. J. Math. Anal. Appl., 467(1):45-66, 2018.

[12] A. Festa and P. Goatin. Modeling the impact of on-line navigation devices in traffic flows. In 2019 IEEE Conference on Decision and Control $(C D C)$, to appear.

[13] M. Garavello and B. Piccoli. Traffic flow on networks, volume 1 of AIMS Series on Applied Mathematics. American Institute of Mathematical Sciences (AIMS), Springfield, MO, 2006. Conservation laws models.

[14] S. K. Godunov. A difference method for numerical calculation of discontinuous solutions of the equations of hydrodynamics. Mat. Sb. (N.S.), 47 (89):271-306, 1959.

[15] G. Guerra and W. Shen. Backward Euler Approximations for Conservation Laws with Discontinuous Flux. arXiv e-prints, page arXiv:1803.00493, Mar 2018.

[16] H. Holden and N. H. Risebro. Front tracking for hyperbolic conservation laws, volume 152 of Applied Mathematical Sciences. Springer, Heidelberg, second edition, 2015.

[17] K. H. Karlsen and J. D. Towers. Convergence of the Lax-Friedrichs scheme and stability for conservation laws with a discontinous space-time dependent flux. Chinese Ann. Math. Ser. B, 25(3):287-318, 2004.

[18] J. Larson, K.-Y. Liang, and K. H. Johansson. A distributed framework for coordinated heavyduty vehicle platooning. IEEE Transactions on Intelligent Transportation Systems, 16(1):419-429, 2014.

[19] C. Lattanzio, A. Maurizi, and B. Piccoli. Moving bottlenecks in car traffic flow: a PDE-ODE coupled model. SIAM J. Math. Anal., 43(1):50-67, 2011.

[20] J.-P. Lebacque. The Godunov scheme and what it means for first order traffic flow models. In Transportation and traffic theory. Proceedings of the 13th international symposium on transportation and traffic theory, Lyon, France, 24-26 JULY 1996, 1996.

[21] J.-P. Lebacque, J.-B. Lesort, and F. Giorgi. Introducing buses into first-order macroscopic traffic flow models. Transportation Research Record, 1644(1):70-79, 1998.

[22] M. J. Lighthill and G. B. Whitham. On Kinematic Waves. II. A Theory of Traffic Flow on Long Crowded Roads. Proceedings of the Royal Society of London. Series A., 229:317-346, 1955.

[23] M. Papageorgiou, I. Papamichail, and Y. Wang. The Role of Macroscopic Modeling in the Simulation, Surveillance and Control of Motorway Network Traffic. In Transport Simulation: Beyond Traditional Approaches, pages 3-25. 2019.

[24] C. Pasquale, S. Sacone, S. Siri, and A. Ferrara. A new Micro-Macro METANET model for platoon control in freeway traffic networks. In Proceedings of the 21st International Conference on Intelligent Transportation Systems (ITSC), pages 1481-1486. IEEE, nov 2018. 
[25] G. Piacentini, P. Goatin, and A. Ferrara. Traffic control via moving bottleneck of coordinated vehicles. Proceedings of 15th IFAC Symposium on Control in Transportation Systems (CTS), 51(9):13-18, jan 2018.

[26] G. Piacentini, C. Pasquale, S. Sacone, S. Siri, and A. Ferrara. Multiple Moving Bottlenecks for Traffic Control in Freeway Systems. In Proceedings of European Control Conference (ECC), 2019.

[27] P. I. Richards. Shockwaves on the highway. Operations Research, 4:42-51, 1956.

[28] C. Roncoli, M. Papageorgiou, and I. Papamichail. Traffic flow optimisation in presence of vehicle automation and communication systems - Part I: A first-order multi-lane model for motorway traffic. Transportation Research Part C: Emerging Technologies, 57:241-259, aug 2015.

[29] A. Talebpour and H. S. Mahmassani. Influence of connected and autonomous vehicles on traffic flow stability and throughput. Transportation Research Part C: Emerging Technologies, 71:143163 , oct 2016 .

[30] J. D. Towers. Convergence of the Godunov scheme for a scalar conservation law with time and space discontinuities. J. Hyperbolic Differ. Equ., 15(2):175-190, 2018.

[31] J. D. Towers. Convergence via OSLC of the Godunov scheme for a scalar conservation law with time and space flux discontinuities. Numer. Math., 139(4):939-969, 2018.

[32] M. Treiber and A. Kesting. Traffic Flow Dynamics: Data, Models and Simulation. Springer-Verlag Berlin Heidelberg, 2013.

[33] S. van de Hoef, K. H. Johansson, and D. V. Dimarogonas. Fuel-efficient en route formation of truck platoons. IEEE Transactions on Intelligent Transportation Systems, 19(1):102-112, 2017.

[34] S. Villa, P. Goatin, and C. Chalons. Moving bottlenecks for the Aw-Rascle-Zhang traffic flow model. Discrete Contin. Dyn. Syst. Ser. B, 22(10):3921-3952, 2017.

[35] M. Zambelli and A. Ferrara. Robustified distributed model predictive control for coherence and energy efficiency-aware platooning. In 2019 American Control Conference (ACC), pages 527-532, July 2019. 